\title{
Abundance determinations with Blackwell diagrams
}

\author{
C. Saffe $\mathrm{S}^{3}$ and H. Levato ${ }^{1,2}$ \\ ${ }^{1}$ Complejo Astronómico El Leoncito, C.C 467, 5400 San Juan, Argentina \\ 2 Member of the Carrera del Investigador Científico, CONICET, Consejo Nacional de Investigaciones Científicas y Técnicas \\ de la República Argentina \\ ${ }^{3}$ Facultad de Ciencias Exactas, Físicas y Naturales, Universidad Nacional de San Juan, Argentina
}

Received 12 May 2003 / Accepted 11 December 2003

\begin{abstract}
We have performed an elemental abundance analysis for Sirius, Vega, and three peculiar silicon stars: HD 43819, HD 192913 and HD 133029 using Blackwell diagrams and observational data taken from the literature. We have compared our results with previous abundance and microturbulence velocity determinations to check the validity of Blackwell diagrams applied to CP stars. We found a very good agreement.
\end{abstract}

Key words. stars: abundances - stars: chemically peculiar

\section{Introduction}

We use the SPECTRUM code spectral synthesis program that permits us to calculate the spectrum synthesis using the fully blanketed stellar atmosphere models of Kurucz (1993) as input. SPECTRUM has been used in several publications ${ }^{1}$. SPECTRUM has a number of auxiliary programs, among them the code BLACKWEL that computes "Blackwell diagrams" (Blackwell \& Shallis 1979) for a given set of spectral lines with measured equivalent widths. Blackwell diagrams are used to determine the elemental abundances and microturbulent velocity for a star, given an appropriate atmosphere model. Blackwell diagrams give the same information as the classical curve of growth method, but without the errors introduced by the many invalid assumptions. BLACKWEL calculates the abundance of the element in question which yields the equivalent width for the given line for a range of microturbulent velocities (which are given as input into BLACKWEL). Then if one plots the abundance versus the microturbulent velocity the result is a sloping line going from high abundances for low microturbulent velocities to low abundances for high microturbulent velocities. Different spectral lines will give differently sloping lines; the intersection (or the region of least confusion) will give the optimal microturbulent velocity for the star and the abundance of the element in question. For best results, one should use isolated lines with well-determined $\log (g f)$ with strengths ranging from weak to medium. Strong lines will not give satisfactory results. Blackwell diagrams have also been used in the past to obtain solar abundances (Blackwell et al. 1995; Blackwell \& Shallis 1979). We want to verify the reliability of this method when applied to normal and chemically

Send offprint requests to: $\mathrm{H}$. Levato,

e-mail: levato@infovia.com.ar

1 See www.phys.appstate.edu/spectrum/spectrum.html peculiar stars. In the present paper we want to compare our results with some available in the literature. We have selected for the comparison elemental abundance determinations for Sirius, Vega and 3 CP stars of the silicon type.

Sirius (Sirius A = HD 48915) has a mild anomalous chemical composition and it has been defined as a mild metallic line star (e.g. Abt 1979), while Vega (=HD 172167) is slightly metal poor with respect to the sun and is now considered to be a mild $\lambda$ Bootis star (e.g. Lemke \& Venn 1996; Ilijic et al. 1998). Many chemical composition studies have been devoted to Sirius (e.g. Sadakane \& Ueta 1989; Hui-Bon-Hoa et al. 1997; Qiu et al. 2001, hereafter QIU) and Vega (e.g. Sadakane \& Nishumira 1979; Adelman \& Gulliver 1990; QIU). We have selected for comparison the abundances derived by QIU for Sirius and Vega, as well as the data sources for $\log g f$ values included by QIU. Also we have adopted the fundamental parameters used by QIU to select the stellar atmosphere models for both stars. We have also applied the method to HD 43819, HD 192913 and HD 133029. They are chemically peculiar (Ap Si) stars, or CP2 in the classification scheme of Preston (1974). Previous elemental abundance determinations have been performed for HD 43819 (López-García \& Adelman 1994, hereafter ZLG1), for HD 192913 and HD 133029 (López-García \& Adelman 1999, hereafter ZLG2). We will adopt the results of these two papers for abundance comparison as well as for the data sources.

The spectra for the CP stars were measured on spectra recorded on nitrogen-baked IIa-O photographic plates and there is no indication of $\mathrm{S} / \mathrm{N}$. The dispersion of the spectra is $4.3 \mathrm{~A} / \mathrm{mm}$, covering approximately $\lambda \lambda 3760-4650$. The data of Sirius and Vega were measured on digital spectra with S/N higher than 300 and resolving power around 40000 , covering approximately $\lambda \lambda 3800-9300$. The two groups of spectra taken from the literature are rather different, and this is one of the 
Table 1. Fundamental parameters.

\begin{tabular}{cccc}
\hline \hline Object & $T_{\text {eff }}[\mathrm{K}]$ & $\log g[\mathrm{dex}]$ & Reference \\
\hline Sirius & $9880 \pm 200$ & $4.40 \pm 0.20$ & QIU \\
Vega & $9430 \pm 200$ & $3.95 \pm 0.20$ & QIU \\
HD 43819 & $11300 \pm 100$ & $3.20 \pm 0.10$ & ZLG1 \\
HD 133029 & $11200 \pm 100$ & $3.84 \pm 0.10$ & ZLG2 \\
HD 192913 & $10900 \pm 100$ & $3.40 \pm 0.10$ & ZLG2 \\
\hline
\end{tabular}

reasons for their selection because we will be able to check if the final abundances have systematic effects depending on the detector used. We could have selected photographic material for the abundance determination of Sirius and Vega but this was not the purpose.

\section{Line identifications and atmospheric parameters}

The observational material, line identifications and selection of lines have been discussed in detail for Sirius and Vega by QIU, for HD 43819 by ZLG1, and for HD 192913 and HD 133029 by ZLG2. Atmospheric parameters have also been estimated for these objects in those papers as listed in Table 1 .

The stellar atmosphere models for Sirius and Vega were generated by QIU, using the ATLAS9 code (Kurucz 1993) assuming LTE, hydrostatic equilibrium and plane parallel stratification. For HD 43819, HD 192913, and HD 133029 ZLG1 and ZLG2 selected a line blanketed ATLAS9 model atmosphere with 10 times the solar metallicity. For proper comparison we have adopted the same stellar atmosphere models.

\section{Abundance analyses}

The abundance determination for Sirius and Vega by QIU were done by comparison of computed equivalent widths with measured values using a code by Magain (1985). The abundance determination for HD 43819 by ZLG1, and for HD 192913 and HD 133029 by ZLG2 were computed using the program WIDTH9 (Kurucz 1992). We redetermined the metal abundances from the equivalent widths with BLACKWEL code for a given set of spectral lines with measured equivalent widths. BLACKWEL calculates for a range of microturbulent velocities $(\zeta)$ the abundance $(A)$ required to reproduce the measured equivalent widths. A plane $\zeta$ vs. $\log A$ is constructed. The abundance for the element and the microturbulence velocity is read from the intersection of the sloping lines which is the point of least confusion (the minimum of the standard deviation for different microturbulent velocities) for each atomic species. BLACKWEL must converge on the correct abundance for a given microturbulent velocity by an iterative process.

As a first step, the microturbulence velocity $\zeta$ is found as the velocity that gives the smallest spread in $\log A$ for a group of lines (see Fig. 1: Standard deviation vs. $\zeta$ ). We present in Table 2 the results for Fe I and Fe II, and for comparison purposes we have also included results for Ti II and $\mathrm{Cr}$ II. The column labeled $\zeta_{1}$ shows the the average microturbulence velocity


Fig. 1. Standard deviation vs. microturbulence $\left[\mathrm{km} \mathrm{s}^{-1}\right]$ for Sirius, Vega, HD 43819, HD 133029 and HD 192913.

Table 2. Derived microturbulence velocities $\left[\mathrm{km} \mathrm{s}^{-1}\right]$ for each specie.

\begin{tabular}{ccccccc}
\hline \hline Object & Ti II & Cr II & Fe I & Fe II & $\zeta_{1}$ & $\zeta_{2}$ \\
\hline Sirius & 2.0 & 2.4 & 1.7 & 1.6 & 1.65 & 1.85 \\
Vega & 2.0 & 2.6 & 1.4 & 1.6 & 1.50 & 1.50 \\
HD 43819 & 0.5 & 1.3 & 0.6 & 1.4 & 1.00 & 0.90 \\
HD 133029 & 1.6 & 2.7 & 2.4 & 2.2 & 2.30 & 2.40 \\
HD 192913 & 1.3 & 1.4 & 1.0 & 1.3 & 1.15 & 0.90 \\
\hline
\end{tabular}

obtained in the present paper from Fe I and Fe II, and the column $\zeta_{2}$ shows the values obtained by ZLG1, ZLG2 and QIU (ZLG1 and ZLG2 calculate $\zeta_{2}$ averaging Fe I and Fe II, while QIU worked directly with Fe I). We found a very good agreement between our $\zeta_{1}$ values and $\zeta_{2}$ values.

Any microturbulence is unexpected if the magnetic Ap stars have quiescient atmospheres as required by various radiative diffusion scenarios (see e.g. Michaud 1970). Alternatively the derived microturbulence is a manifestation of an organized magnetic field. Each line has its own effective microturbulence due to the width of its Zeeman pattern and the distribution of its components. 

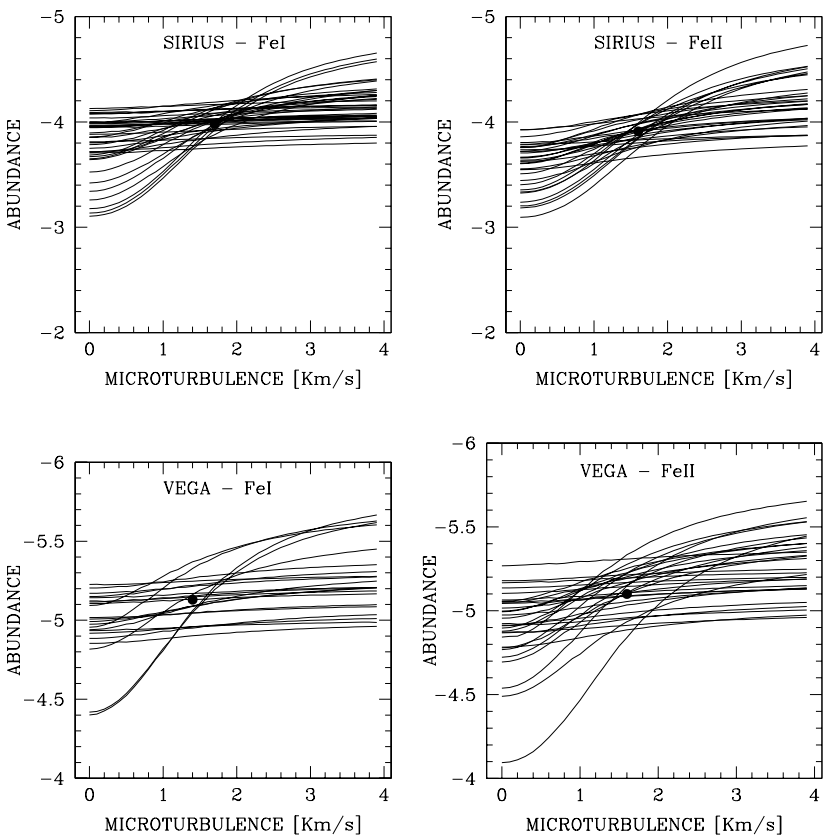

Fig. 2. Fe II and Fe I Blackwell diagrams for Sirius and Vega, i.e. Abundance vs. Microturbulence velocity. Each curve in the diagram represent a single line in the spectra. The point in each diagram represents the minimum confusion region in the $(\log A, \zeta)$ plane and is there where the abundance and microturbulence velocity is read off for each species (see Table 2).

As an example of the calculation, Fig. 2 shows the Blackwell diagrams for Sirius and Vega from Fe I and Fe II lines. The points of least confusion correspond to the minima of Fig. 1.

The final test for the derived microturbulence velocities will be the exploration of any dependence between the derived abundances and the equivalent widths. As an example, Fig. 3 shows the Fe II abundance vs. equivalent widths for HD 133029, HD 192913, HD 43819, Sirius and Vega. The major absolute value of the slope is $4.710 \mathrm{E}-3$ for Vega's Fe I, derived with 22 lines. This implies a difference of 0.37 dex in abundances for two points separated by $80 \mathrm{~m} \AA$ (the maximum separation between two points for Vega's Fe I).

Once we have determined the microturbulence velocity $\zeta_{1}$, we recalculated the abundances of all species using this value. In Tables 3 and 4 we present the derived abundances for the five stars for each atomic species, the standard deviation and the number of lines used per element. The column labeled $\Delta$ shows the difference between our abundance values and those obtained by ZLG1 (HD 43819), ZLG2 (HD 133029, HD 192913) and QIU (Sirius, Vega).

\section{Discussion}

Figure 4 shows the abundance determination using Blackwell diagrams ( $y$ axis) vs. abundance determination from ZLG1, ZLG2 and QIU ( $x$ axis), i.e. the graphical representation of Tables 3 and 4 . We have calculated for each star a straight line
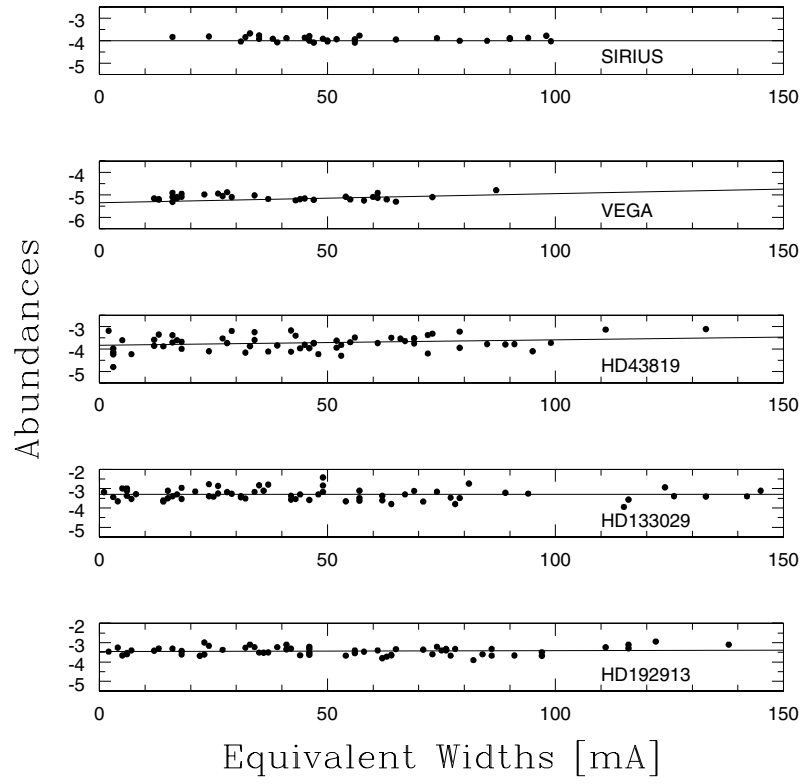

Fig.3. Abundance vs. equivalent width for Sirius, Vega, HD 43819, HD 133029 and HD 192913 respectively.

Table 3. Derived abundances for Sirius and Vega.

\begin{tabular}{|c|c|c|c|c|c|c|}
\hline & Sirius & & & Vega & & \\
\hline Species & $\log \mathrm{N} / \mathrm{H}$ & $n$ & $\Delta$ & $\log \mathrm{N} / \mathrm{H}$ & $n$ & $\Delta$ \\
\hline C I & $-4.49 \pm 0.13$ & 2 & -0.09 & $-3.60 \pm 0.13$ & 3 & -0.02 \\
\hline N I & $-3.88 \pm 0.17$ & 7 & 0.12 & $-3.85 \pm 0.14$ & 2 & 0.19 \\
\hline O I & $-3.46 \pm 0.21$ & 2 & -0.05 & $-2.92 \pm 0.15$ & 4 & 0.11 \\
\hline $\mathrm{Na} \mathrm{I}$ & $-4.34 \pm 0.00$ & 1 & -0.17 & $-5.63 \pm 0.00$ & 1 & -0.04 \\
\hline Mg I & $-4.93 \pm 0.62$ & 3 & -0.33 & $-5.25 \pm 0.00$ & 1 & -0.02 \\
\hline $\mathrm{Mg}$ II & $-4.34 \pm 0.00$ & 1 & 0.03 & $-5.07 \pm 0.01$ & 2 & 0.29 \\
\hline $\mathrm{Al} \mathrm{I}$ & $-5.36 \pm 0.00$ & 1 & 0.08 & $\ldots \ldots$ & & $\ldots$ \\
\hline $\mathrm{Al}$ II & $-5.35 \pm 0.25$ & 2 & -0.07 & $\ldots \ldots$ & & $\ldots$ \\
\hline Si II & $-4.05 \pm 0.05$ & 3 & 0.01 & $-5.02 \pm 0.07$ & 3 & 0.06 \\
\hline S II & $-4.75 \pm 0.00$ & 1 & 0.15 & $\ldots \ldots$ & & $\ldots$ \\
\hline $\mathrm{Ca} \mathrm{I}$ & $-6.18 \pm 0.04$ & 2 & 0.47 & $-6.31 \pm 0.10$ & 4 & 0.32 \\
\hline $\mathrm{Ca}$ II & $-6.71 \pm 0.00$ & 1 & -0.35 & $-6.58 \pm 0.00$ & 1 & 0.44 \\
\hline Sc II & $-9.91 \pm 0.00$ & 1 & 0.47 & $-9.94 \pm 0.31$ & 2 & -0.23 \\
\hline Ti II & $-6.57 \pm 0.19$ & 33 & 0.20 & $-7.44 \pm 0.12$ & 17 & -0.03 \\
\hline V II & $-7.32 \pm 0.11$ & 3 & 0.19 & $-8.27 \pm 0.00$ & 1 & 0.03 \\
\hline $\mathrm{Cr} \mathrm{I}$ & $-5.51 \pm 0.31$ & 5 & 0.19 & $\ldots \ldots$ & & $\ldots$ \\
\hline Cr II & $-5.58 \pm 0.15$ & 19 & 0.16 & $-6.81 \pm 0.17$ & 14 & 0.04 \\
\hline Mn I & $-6.34 \pm 0.06$ & 2 & 0.06 & $-6.97 \pm 0.11$ & 2 & -0.02 \\
\hline $\mathrm{Fe} \mathrm{I}$ & $-3.98 \pm 0.12$ & 64 & 0.05 & $-5.13 \pm 0.10$ & 22 & -0.03 \\
\hline Fe II & $-3.91 \pm 0.13$ & 34 & 0.13 & $-5.10 \pm 0.10$ & 33 & 0.01 \\
\hline Co I & $-5.42 \pm 0.00$ & 1 & 0.04 & $\ldots \ldots$ & & $\ldots$ \\
\hline Ni I & $-4.94 \pm 0.13$ & 18 & 0.00 & $5.80 \pm 0.00$ & 1 & -0.03 \\
\hline Ni II & $-4.87 \pm 0.05$ & 3 & 0.12 & $\ldots \ldots$ & & $\ldots$ \\
\hline $\mathrm{Zn} \mathrm{I}$ & $-6.31 \pm 0.00$ & 1 & 0.04 & $\ldots \ldots$ & & $\ldots$ \\
\hline Sr II & $-8.51 \pm 0.05$ & 2 & 0.33 & $-10.40 \pm 0.05$ & 2 & 0.36 \\
\hline Y II & $-8.98 \pm 0.04$ & 3 & 0.41 & $-10.19 \pm 0.19$ & 2 & 0.20 \\
\hline $\mathrm{Zr}$ II & $-8.28 \pm 0.06$ & 3 & 0.26 & $-10.23 \pm 0.00$ & 1 & 0.14 \\
\hline $\mathrm{Ba}$ II & $-8.99 \pm 0.27$ & 3 & 0.29 & $-10.79 \pm 0.27$ & 1 & 0.44 \\
\hline La II & $-9.65 \pm 0.00$ & 1 & 0.51 & $-10.55 \pm 0.00$ & 1 & 0.41 \\
\hline
\end{tabular}


Table 4. Derived abundances for HD 43819, HD 133029 and HD 192913.

\begin{tabular}{|c|c|c|c|c|c|c|c|c|c|}
\hline & HD 43819 & & & HD 133029 & & & HD 192913 & & \\
\hline Species & $\log \mathrm{N} / \mathrm{H}$ & $n$ & $\Delta$ & $\log \mathrm{N} / \mathrm{H}$ & $n$ & $\Delta$ & $\log \mathrm{N} / \mathrm{H}$ & $n$ & $\Delta$ \\
\hline C II & $-3.80 \pm 0.12$ & 2 & -0.02 & $-3.34 \pm 0.00$ & 1 & 0.32 & $-2.92 \pm 0.44$ & 2 & 0.24 \\
\hline Mg I & $-4.75 \pm 0.20$ & 2 & 0.27 & $-5.33 \pm 0.25$ & 3 & -0.29 & $-5.17 \pm 0.26$ & 2 & -0.07 \\
\hline Mg II & $-4.71 \pm 0.02$ & 4 & 0.01 & $-4.39 \pm 0.11$ & 5 & -0.06 & $-4.69 \pm 0.20$ & 6 & 0.01 \\
\hline $\mathrm{Al} \mathrm{I}$ & $\ldots \ldots$ & & $\ldots$ & $\ldots \ldots$ & & $\ldots$ & $-5.39 \pm 0.14$ & 2 & -0.06 \\
\hline Si II & $-4.11 \pm 0.19$ & 5 & -0.23 & $-3.69 \pm 0.07$ & 5 & -0.12 & $-4.14 \pm 0.19$ & 5 & -0.08 \\
\hline Si III & $\ldots \ldots$ & & $\ldots$ & $-3.10 \pm 0.20$ & 3 & 0.12 & $\ldots \ldots$ & & $\ldots$ \\
\hline S II & $-5.57 \pm 0.07$ & 3 & -0.11 & $-4.51 \pm 0.36$ & 4 & 0.16 & $-4.68 \pm 0.21$ & 3 & 0.16 \\
\hline Ca II & $-5.33 \pm 0.00$ & 1 & -0.21 & $-5.94 \pm 0.00$ & 1 & -0.61 & $-5.50 \pm 0.00$ & 1 & 0.24 \\
\hline Sc II & $-8.81 \pm 0.00$ & 1 & 0.30 & $\ldots \ldots$ & & $\ldots$ & $-8.74 \pm 0.36$ & 1 & -0.05 \\
\hline Ti II & $-5.69 \pm 0.26$ & 45 & 0.12 & $-6.15 \pm 0.33$ & 31 & -0.13 & $-5.67 \pm 0.25$ & 45 & -0.07 \\
\hline Cr I & $-4.60 \pm 0.00$ & 1 & 0.09 & $-4.13 \pm 0.24$ & 9 & -0.29 & $-3.76 \pm 0.00$ & 1 & -0.16 \\
\hline Cr II & $-5.02 \pm 0.25$ & 28 & 0.00 & $-4.17 \pm 0.34$ & 46 & -0.02 & $-4.99 \pm 0.21$ & 36 & -0.07 \\
\hline Mn II & $-5.65 \pm 0.45$ & 4 & -0.09 & $-5.29 \pm 0.30$ & 10 & -0.23 & $-4.97 \pm 0.33$ & 11 & -0.21 \\
\hline $\mathrm{Fe} \mathrm{I}$ & $-3.30 \pm 0.30$ & 55 & 0.18 & $-3.57 \pm 0.25$ & 39 & -0.25 & $-3.36 \pm 0.19$ & 51 & -0.10 \\
\hline $\mathrm{Fe}$ II & $-3.73 \pm 0.30$ & 68 & -0.07 & $-3.27 \pm 0.29$ & 69 & 0.01 & $-3.37 \pm 0.21$ & 68 & -0.01 \\
\hline $\mathrm{Fe}$ III & $-3.85 \pm 0.00$ & 1 & -0.15 & $-3.17 \pm 0.37$ & 5 & 0.01 & $-2.79 \pm 0.25$ & 4 & -0.11 \\
\hline Ni II & $-6.56 \pm 0.00$ & 1 & -0.07 & $-5.73 \pm 0.28$ & 4 & -0.03 & $-5.31 \pm 0.33$ & 4 & -0.05 \\
\hline Sr II & $-5.62 \pm 0.39$ & 3 & 0.01 & $-7.46 \pm 0.10$ & 2 & -0.45 & $-5.10 \pm 0.36$ & 4 & -0.07 \\
\hline Y II & $\ldots \ldots$ & & $\ldots$ & $-8.12 \pm 0.19$ & 5 & -0.33 & $-7.76 \pm 0.14$ & 4 & -0.17 \\
\hline $\mathrm{Zr}$ II & $-7.71 \pm 0.28$ & 3 & 0.10 & $-7.93 \pm 0.23$ & 2 & -0.23 & $-7.30 \pm 0.34$ & 7 & -0.08 \\
\hline Ba II & $\ldots \ldots$ & & $\ldots$ & $-8.92 \pm 0.00$ & 1 & -0.24 & $-8.58 \pm 0.00$ & 1 & -0.06 \\
\hline Ce II & $-6.74 \pm 0.33$ & 8 & 0.12 & $-7.32 \pm 0.22$ & 14 & -0.18 & $-6.87 \pm 0.27$ & 25 & -0.01 \\
\hline Pr II & $-6.34 \pm 0.00$ & 1 & 0.35 & $-7.06 \pm 0.17$ & 6 & -0.08 & $-6.81 \pm 0.18$ & 4 & -0.15 \\
\hline Nd II & $-7.22 \pm 0.00$ & 1 & 0.12 & $-6.96 \pm 0.12$ & 5 & -0.26 & $-6.89 \pm 0.27$ & 6 & -0.08 \\
\hline Sm II & $\ldots \ldots$ & & $\ldots$ & $\ldots \ldots$ & & $\ldots$ & $-6.46 \pm 0.19$ & 12 & -0.12 \\
\hline $\mathrm{Eu}$ II & $-8.03 \pm 0.12$ & 3 & 0.12 & $-8.72 \pm 0.21$ & 4 & -0.28 & $-5.43 \pm 0.38$ & 4 & -0.14 \\
\hline Gd II & $-7.05 \pm 0.41$ & 2 & 0.13 & $-7.54 \pm 0.35$ & 11 & -0.30 & $-7.52 \pm 0.41$ & 4 & -0.08 \\
\hline Dy II & $\ldots \ldots$ & & $\ldots$ & $\ldots \ldots$ & & $\ldots$ & $-6.42 \pm 0.25$ & 6 & 0.14 \\
\hline $\mathrm{Hg}$ II & $\ldots \ldots$ & & $\ldots$ & $-6.51 \pm 0.00$ & 1 & 0.06 & $-5.30 \pm 0.00$ & 1 & -0.18 \\
\hline
\end{tabular}

that fits the data (the equation of this line is presented in the figures). From Tables 3 and 4 it is clear that the major differences between the abundances obtained by ZLG1, ZLG2, and QIU and those derived by the Blackwell diagrams occur for Ca II, Sr II, C II, Sc II, Pr II, Ca I, La II, and Ba II whose abundances are based on very few lines. The value of these discrepancies are between 0.2 and 0.6 dex. We conclude that there is a general agreement between the abundances for the different atomic species using the Blackwell diagrams and other abundance determination methods using Kurucz classical models in LTE.

Acknowledgements. The present work was partially supported through a grant from the Consejo Nacional de Investigaciones Científicas y Técnicas, República Argentina PIP 2124. 

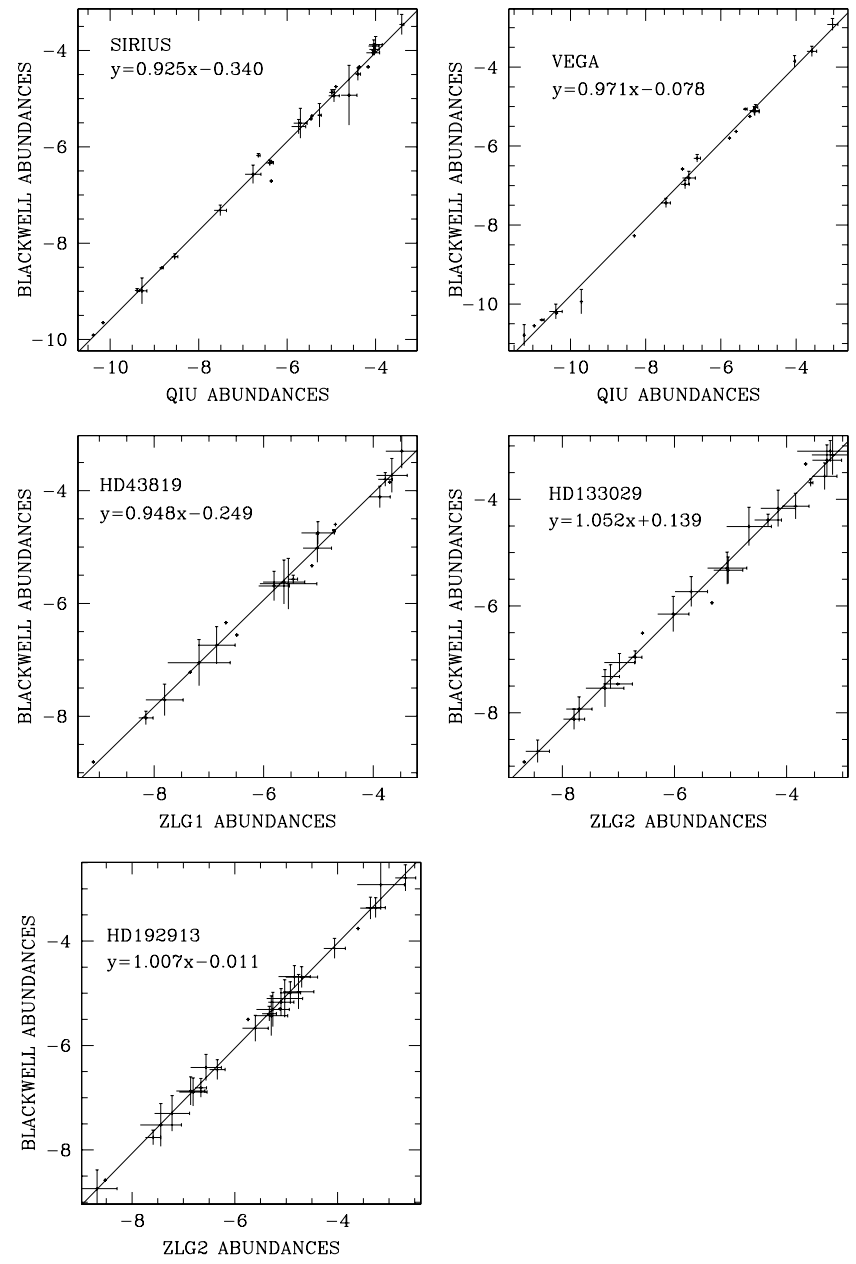

Fig. 4. Comparison of QIU vs. Blackwell diagram abundances for Sirius and Vega, ZLG1 vs. Blackwell diagram for HD 43819, ZLG2 vs. Blackwell diagram for HD 133129 and HD 192913. The error bar are the standard deviation for each species. The fitted straight line coefficients are shown.

\section{References}

Abt, H. A. 1979, ApJ, 230, 485

Adelman, S. J., \& Gulliver, A. F. 1990, ApJ, 348, 712

Blackwell, D. E., \& Shallis, M. J. 1979, MNRAS, 186, 673

Blackwell, D. E., Lynas-Gray, A. E., \& Smith, G. 1994, A\&A, 296, 217

Hui-Bon-Hoa, A., Burkhart, C., \& Alecian, G. 1997, A\&A, 323, 901

Ilijic, S., Rosandic, M., Planicic, M., \& Pavlovski, K. 1998, Coska, 27,461

Kurucz, R. L. 1993, CD-ROM 13 (Cambridge: SAO)

Kurucz, R. L., Private communication

Lambert, D. L., Roby, S. W., \& Bell, R. A. 1982, ApJ, 254, 663

Lemke, M., \& Venn, K. A. 1996, A\&A, 309, 558

Magain, P. 1985, A\&A, 146, 95

Michaud, G. 1970, ApJ, 160, 641

Preston, G. W. 1974, ARA\&A, 12, 297

Qiu, H. M., Zhao, G., Chen, Y. Q., \& Li, Z. W. 2001, ApJ, 548, 953

Sadakane, K. 1991, PASP, 103, 355

Sadakane, K., Jugaku, J., \& Takada-Hidai, M. 1988, PASP, 100, 811

Sadakane, K., \& Nishimura, M. 1979, PASJ, 31, 481

Sadakane, K., \& Ueta, M. 1989, PASJ, 41, 279

Lopez-Garcia, Z., \& Adelman, S. J. 1994, A\&AS, 107, 353

Lopez-Garcia, Z., \& Adelman, S. J. 1999, A\&AS, 137, 227 